\title{
Du droit souple au droit dur : quel droit pour la qualité environnementale des vins sous indication géographique?
}

\section{From soft law to hard law: What kind of legislation for environmental quality of wines produced under geographical indication?}

\author{
R. Raffray
}

Professeur de droit privé, Université de Bordeaux, Institut des sciences de la vigne et du vin, Institut de recherche en droit des affaires et du patrimoine, Avenue Léon Duguit, 33608 Pessac, France

\begin{abstract}
Résumé. Le droit du vin accompagne la réception par la filière viticole des valeurs de la Responsabilité Sociale et Environnementale. Les producteurs de vin et les pouvoirs publics recherchent un modèle plus éthique, plus performant sur le plan environnemental, compatible avec le développement durable. Les instruments juridiques utilisés ont beaucoup évolué en vingt ans. Dans un premier temps, les producteurs ont eu recours à des instruments volontaires, non contraignants, caractéristiques du droit souple (chartes éthiques, certification biologique, normes ISO). Une nouvelle règlementation est apparue en droit français. La loi prévoit, d'ici 2030, une certification environnementale, rendue obligatoire par le cahier des charges, pour tout producteur viticole exploitant sous signe de qualité (Appellation d'Origine Protégée ; Indication Géographique Protégée). Ce passage du droit volontaire (souple) au droit imposé (droit dur) est significatif de l'évolution fondamentale de la notion de qualité viticole, qui intègre la qualité environnementale. La qualité environnementale enrichit la qualité liée à l'origine.
\end{abstract}

\begin{abstract}
Wine law accompanies the reception by the wine industry of the values of the Social and Environmental Responsibility. Wine producers and public authorities are looking for a more ethical, more environmentally-friendly model that is compatible with sustainable development. The legal instruments used have evolved considerably in twenty years. As a first step, producers have used voluntary instruments, which are characteristic of flexible law (ethical charters, organic certification, ISO standards). A new regulation appeared in French law. Law provides, by 2030, an environmental certification, made mandatory by the specifications, for any wine producer operating under sign of quality (protected designation of origin and protected geographical indication). This transition from voluntary law (flexible law) to imposed law (hard law) is significant of the evolution of the legal concept of wine quality, which integrates environmental quality. These legal evolutions illustrate the fundamental evolution of the notion of quality: environmental quality enriches the quality linked to origin.
\end{abstract}

« La soft law poursuit la même ambition d'orientation des conduites que la hard law. Seulement, là où la hard law impose, la soft law propose. Le droit souple veut utiliser le ressort de l'incitation et de l'adhésion, en vue d'obtenir les conduites par la douceur et non par la punition» [P. Deumier, Introduction au droit, $\mathbf{n}^{\circ} \mathbf{3 0}$ (LGDJ, 4 ${ }^{\text {ème }}$ éd., 2017)]. L'accompagnement juridique des progrès environnementaux dans la production de vins sous indication géographique connaît une modification en profondeur en France. A l'origine laissées à l'appréciation des viticulteurs, et règlementées par des dispositifs apparentés au droit souple, des démarches environnementales vont dans un avenir proche, et dans une mesure modeste, s'imposer aux viticulteurs sur le modèle d'un droit contraignant. Il s'agit d'une période charnière pour le droit viticole, qui doit accompagner au mieux la filière dans sa révolution environnementale sans bouleverser les équilibres construits par la législation viticole.

Dans un entretien paru en 2013 [Entretien avec P. Philippe, Directeur de la société coopérative des vignerons de Buzet, in Olivier Antoine-Geny et Marcello Roudil, Manuel pratique du commerce des vins, p. 28 et s. (Féret, 2013)], le directeur d'une coopérative viticole ayant axé sa stratégie sur une viticulture différenciée décrivait de la manière suivante le consommateur de la décennie qui s'annonçait: «Un consommateur plus responsable, dont l'acte d'achat sera plus engagé. Je ne crois pas pour autant au «tout bio». Simplement, je pense avec conviction que ce nouveau consommateur sera plus vigilant sur la nature durable des produits que nous lui proposerons. Il voudra des vins, certes 
bons pour son goût, mais aussi sans risque pour sa santé, dont les pratiques de production seront respectueuses de l'environnement, et peut-être même dont les conditionnements seront recyclables... bref, un consommateur aux tendances «alter» $\gg$. Comme tout autre observateur de la viticulture, le juriste est confronté à un phénomène d'ampleur qui bouscule ses représentations fondamentales. Il s'agit de la multiplication des démarches «éthiques» dans la filière viticole [sur lesquelles v. R. Raffray (dir.) L'éthique et le vin, le droit comme levier d'une nouvelle qualité, Droit et Patrimoine, (Dossier, juin 2018)]. Lorsque l'on parle d'éthique en matière viticole, on évoque, classiquement, un modèle de production, respectueux du terroir, de la tradition, et tourné vers la recherche de qualité, valorisé collectivement en France et dans l'Union européenne par le système de l'appellation d'origine. Cependant, pour le juriste, dans une signification plus contemporaine, l'éthique, dans la filière viticole, renvoie plutôt à une démarche individuelle pour celui qui souhaite produire dans des conditions sociales et environnementales acceptables. C'est cette éthique-là qui s'est appuyée sur les valeurs et les démarches de la Responsabilité Sociale et Environnementale (RSE) pour que naisse un modèle de viticulture compatible avec le développement durable.

La responsabilité sociale et environnementale a été conceptualisée aux Etats-Unis mais concrétisée en Europe, notamment sous l'impulsion de la Commission Européenne qui la définissait en 2001 comme «l'intégration volontaire des préoccupations sociales et écologiques des entreprises à leurs activités commerciales et leurs relations avec les parties prenantes. Etre socialement responsable signifie non seulement satisfaire pleinement aux obligations juridiques applicables, mais aussi aller au-delà et investir «davantage» dans le capital humain, l'environnement et les relations avec les parties prenantes» [Commission Européenne, «Promouvoir un cadre européen pour la responsabilité sociale des entreprises» Livre vert de la Responsabilité Sociale des Entreprises» (2001)]. Ici apparaît le terme de «parties prenantes», terme utilisé à l'origine par la science économique pour distinguer, dans les grandes sociétés, les share holders (les actionnaires), et les stake holders, (les parties prenantes), individu, groupe ou valeur qui peut affecter ou être affecté par la réalisation des objectifs organisationnels (les actionnaires, salariés, dirigeants, fournisseurs, clients, banques, pouvoirs publics, droits fondamentaux, environnement, associations, communautés locales etc.). Cette définition de la RSE a été remplacée, au niveau de la Commission européenne, par une définition plus synthétique ne faisant plus référence au caractère volontaire de la démarche. Il s'agit de «la responsabilité des entreprises vis-à-vis des effets qu'elles exercent sur la société» [Responsabilité sociale des entreprises: une nouvelle stratégie de l'UE pour la période 2011-2014, Communication de la Commission au Parlement européen, au Conseil, au Comité économique et social européen et au Conseil des régions.] Pour l'International Organisation for Standardisation (ISO), elle doit être définie à la rencontre de l'éthique, des parties prenantes du développement durable comme «la responsabilité d'une organisation vis-à-vis des impacts de ses décisions et activités sur la société et sur l'environnement, se traduisant par un comportement éthique et transparent qui contribue au développement durable, y compris à la santé et au bien-être de la société ; prend en compte les attentes des parties prenantes; respecte les lois en vigueur et qui est en accord avec les normes internationales de comportement; et qui est intégré dans l'ensemble de l'organisation et mis en ouvre dans ses relations》.

La démarche RSE est aujourd'hui perçue comme une donnée et un levier de compétitivité par de nombreuses entreprises à tel point que sont devenus familiers les nouveaux indicateurs de calcul de la triple bottom line (people, planet, profit) permettant une évaluation sociale, environnementale et économique de l'entreprise. La RSE est également apparue comme un moyen privilégié de contribuer au développement durable, défini dans le rapport Brundtland comme un « mode de développement qui répond aux besoins des générations présentes sans compromettre la capacité des générations futures de répondre aux leurs».

En France le droit rural n'est pas spécialement orienté vers le développement durable [v. sur cette question P.-E. Bouillot, Le droit face aux enjeux de l'agriculture durable, préf. F. Collart-Dutilleul, Cosmografia, 2017]. Si le droit de l'espace rural s'y réfère expressément [CRPM, art. L. 111-1], la dimension sociale des règles d'accès à la terre est confinée à la protection du preneur et du modèle familial de l'entreprise agricole. La réception des enjeux environnementaux s'est, elle, essentiellement réalisée via les baux ruraux à clauses environnementales. Quant au droit des activités agricoles, il s'y réfère implicitement mais les objectifs environnementaux sont généralement considérés comme accessoires. Il est pourtant admis que la matière agricole dispose de leviers intéressants que sont les labels (entendus largement, tels que les signes de qualité et de l'origine et les marques collectives), car ils permettent de soutenir les prix et de valoriser les efforts accomplis, mais l'on s'interroge sur les limites du caractère volontaire des démarches [sur ces limites, v. P.-E. Bouillot, ouvrage préc., $n^{\circ} 206$ et s.].

Précisément, cette propagation du progrès environnemental connaît actuellement, en France, une nouvelle approche par les pouvoirs publics, soucieux d'imposer de nouvelles normes pour généraliser les progrès environnementaux en agriculture, pour les productions sous signe de qualité et de l'origine, ce qui expose tout particulièrement la viticulture [LOI $n^{\circ} 2018-938$ du 30 octobre 2018 pour l'équilibre des relations commerciales dans le secteur agricole et alimentaire et une alimentation saine, durable et accessible à tous, JORF n 0253 (1 novembre 2018). Sur la loi v. RD rur., $\mathbf{n}^{\circ}$ 5472, Dossier $\mathrm{n}^{\circ} 24$, p. 7 (avril 2019) et $\mathrm{s}$. Sur la certification environnementale, v. spécialement R. Raffray, «Expression et diffusion de l'agroécologie : certification environnementale obligatoire pour les signes d'identification de la qualité et de l'origine» dans le même dossier]. Dans cette transition entre droit souple et droit dur, face à la multiplication des normes, le juriste se présente aujourd'hui comme un levier incontournable de développement, ce qui le replace au centre de l'entreprise. Pour agir au plus près des besoins de la filière et des exigences nouvelles des pouvoirs publics le juriste doit s'attacher au contexte de son intervention (1), ce qui lui permettra de comprendre au mieux ce passage du droit souple au droit dur pour accompagner les 
progrès environnementaux de la viticulture (2) tout en s'interrogeant sur les conséquences de ce mouvement quant à la notion de qualité (3).

\section{Le contexte}

\subsection{Le contexte politique}

Les valeurs de la RSE se retrouvent dès les premières dispositions du Code rural et de la pêche maritime. L'article premier du Livre préliminaire aux dispositions législatives dispose que «I.- La politique en faveur de l'agriculture et de l'alimentation, dans ses dimensions internationale, européenne, nationale et territoriale, a pour finalités: $1^{\circ}$ Dans le cadre de la politique de l'alimentation définie par le Gouvernement, d'assurer à la population l'accès à une alimentation sûre, saine, diversifiée, de bonne qualité et en quantité suffisante, produite dans des conditions économiquement et socialement acceptables par tous, favorisant l'emploi, la protection de l'environnement et des paysages et contribuant à l'atténuation et à l'adaptation aux effets $d u$ changement climatique». Elles se retrouvent exprimées dans la notion d'agroécologie, devenue l'un des supports du développement durable en agriculture.

L'agroécologie est en effet inscrite dans le Livre préliminaire aux dispositions législatives du Code rural et de la pêche maritime. L'article L1-II dispose que : «Les politiques publiques visent à promouvoir et à pérenniser les systèmes de production agroécologiques, dont le mode de production biologique, qui combinent performance économique, sociale, notamment à travers un haut niveau de protection sociale environnementale et sanitaire. Ces systèmes privilégient l'autonomie des exploitations agricoles et l'amélioration de leur compétitivité, en maintenant ou en augmentant la rentabilité économique, en améliorant la valeur ajoutée des productions et en réduisant la consommation d'énergie, d'eau, d'engrais, de produits phytopharmaceutiques et de médicaments vétérinaires, en particulier les antibiotiques. Ils sont fondés sur les interactions biologiques et l'utilisation des services écosystémiques et des potentiels offerts par les ressources naturelles, en particulier les ressources en eau, la biodiversité, la photosynthèse, les sols et l'air, en maintenant leur capacité de renouvellement du point de vue qualitatif et quantitatif. Ils contribuent à l'atténuation et à l'adaptation aux effets du changement climatique». L'agroécologie est définie, selon le Ministère de l'agriculture, comme «une façon de concevoir des systèmes de production qui s'appuient sur les fonctionnalités offertes par les écosystèmes en les amplifiant tout en visant à diminuer les pressions sur l'environnement et à préserver les ressources naturelles». Il s'agit alors «d'utiliser au maximum la nature comme facteur de production en maintenant ses capacités de renouvellement». L'agroécologie implique ainsi «le recours à un ensemble de techniques qui considèrent l'exploitation agricole dans son ensemble. C'est grâce à cette approche systémique que les résultats techniques et économiques peuvent être maintenus ou améliorés tout en améliorant les performances environnementales».

En faisant appel aux capacités de renouvellement, l'agroécologie puise aux idées fondatrices du développement durable dont elle apparaît, tout comme l'agriculture biologique, comme l'un des vecteurs potentiels et essentiels. Son support juridique constitue donc un enjeu majeur. L'agroécologie s'appuie fortement sur les démarches volontaires des agriculteurs, ce dont atteste la multiplication des signes et labels [sur la notion de label agricole, v. D. Rochard, J.-Cl. Rural, Fasc. 20: «Qualité des produits. - Labels et certifications de conformité» ; P.-E. Bouillot, ouvrage préc., p. 177 et s. V. aussi J.-P. Combenègre, Les signes de qualité, (Editions France Agricole, $2^{\text {ème }}$ éd., 2015)]. Si leur foisonnement apporte la preuve de la vitalité des démarches environnementales spontanées en viticulture, elle rend aussi confuse l'identification de ces démarches par le consommateur lorsqu'elles se placent dans le vaste interstice entre l'agriculture conventionnelle et l'agriculture biologique. A l'heure actuelle les pouvoirs publics français cherchent donc à tracer une voie médiane entre ces deux agricultures, avec pour objectif économique qu'en 2030 un tiers de la production agricole soit faite en agriculture biologique, un tiers certification «Haute Valeur Environnementale», la production agroindustrielle et d'exportation demeurant conventionnelle pour le dernier tiers.

En envisageant l'exploitation agricole dans son ensemble, ce qui implique de considérer non seulement l'activité de production mais également l'ensemble des aspects de l'entreprise, l'agroécologie rejoint également la RSE qui a trouvé sa place en viticulture sous de multiples formes, [v. notamment R. Raffray, «Une RSE innommée au service des démarches éthiques de la filière vin», in $\mathrm{R}$. Raffray (dir.), L'éthique et le vin. Le droit comme levier d'une nouvelle qualité ? Dr. et Patr., $\mathbf{n}^{\circ}$ 281, p. 30 et s (2018)].

\subsection{Le contexte organisationnel}

La taille des entreprises viticoles françaises de production ne les destine pas nécessairement à entreprendre des démarches prioritairement conçues pour les grandes entreprises. Pourtant, le caractère collectif de la production s'y prête, par exemple par l'entremise d'associations de producteurs (vignerons indépendants, vins naturels) ou de caves coopératives de production. L'intervention agro écologique peut aussi se décider au niveau de l'appellation [pour les missions de l'ODG, v. CRPM, art. L. 622-17 et s., l'article L. 622-17 évoquant par exemple «la mission d'intérêt général de préservation et de mise en valeur des terroirs»] et trouver un appui dans l'interprofession [v. notamment CRPM, art. L. 632-1, $7^{\circ}$, selon lequel les interprofessions doivent « favoriser les démarches collectives visant à prévenir et à gérer les risques et aléas liés à la production, à la transformation, à la commercialisation et à la distribution des produits agricoles et alimentaires, notamment les aléas et risques sanitaires, phytosanitaires et environnementaux»].

\subsection{Le contexte idéologique}

La RSE promeut un certain nombre de valeurs sociales, environnementales, de garantie des droits de l'homme et de protection des consommateurs. En matière viticole la préoccupation environnementale est érigée en valeur première. Les préoccupations sociales ne sont pas absentes de ce nouveau champ investi par la filière, en raison 
de la nécessité de protéger la santé des viticulteurs [A. Charbonneau, «Compliance et santé au travail dans la viticulture», in R. Raffray (dir.) L'éthique et le vin. Le droit comme levier d'une nouvelle qualité ? Dr. et Patr. $\mathbf{n}^{\circ}$ 281, p. 52 et s. (2018)], de garantir des revenus décents, de tenir compte de la pénibilité et de la précarité-notamment lorsqu'il est saisonnier-du travail viticole. La santé et la sécurité des consommateurs deviennent aussi un champ de RSE viticole, jusque dans l'acte de consommation, la filière souhaitant aujourd'hui promouvoir une consommation responsable de qualité. Au sein des parties prenantes les voisins deviennent aussi un enjeu tout particulier [v. infra].

A côté de ces valeurs communes à toutes les démarches RSE des valeurs propres au secteur peuvent se développer, sur une base ancienne, au soutien du terroir. Ainsi sont pris des engagements qui agissent comme leviers de protection de l'un des éléments du terroir, le paysage et le patrimoine architectural, dans le but d'accompagner l'œnotourisme ou encore les chartes associatives, comme celle des vignerons indépendants, qui mettent en avant le travail du vigneron dans sa dimension individuelle. On trouve enfin les chartes des associations de producteurs de vins naturels, tournée vers la pureté et un certain dépouillement du produit, qui fait écho à l'opposition entre vin artificiel et naturel de la période charnière que fut la fin du $19^{\text {ème }}$ siècle. On se confrontera aussi avec intérêt à l'émergence de valeurs nouvelles comme le lien direct entre le producteur et le consommateur, l'économie collaborative, sociale, solidaire, le financement participatif et l'innovation, telles que présentés sur les plateformes de financement participatif [A. Bienvenu et R. Raffray. «Crowdfunding et vin» in M. Villemonteix (dir.), Le crowdfunding (ou financement participatif), Droit et Pat. $\mathbf{n}^{\circ}$ 270, p. 47 et s. (juin 2017) ]. On notera aussi la volonté affichée de « casser les codes», expression qui renvoie au phénomène d'ubérisation et exprime une certaine défiance à l'égard du système protecteur mais donc rigide de l'appellation d'origine et des infrastructures traditionnelles du négoce.

\section{Les dispositifs}

\subsection{Les dispositifs de droit souple}

En matière viticole, et plus largement même en matière agricole, le développement durable s'est appuyé sur le droit souple. Mais de quoi parle-t-on? Les normes de droit souple répondent à trois conditions cumulatives : elles ont pour objet de modifier ou d'orienter les comportements de leurs destinataires en suscitant, dans la mesure du possible, leur adhésion ; elles ne créent pas par elles-mêmes de droits ou d'obligations pour leurs destinataires ; elles présentent, par leur contenu et leur mode d'élaboration, un degré de formalisation et de structuration qui les apparente aux règles de droit [Conseil d'état, Le droit souple, Volume 64 (étude annuelle 2013) La documentation française, p. 86 et s., spéc. p. 9]. De prime abord, une telle norme paraît inadaptée à la matière viticole, production agricole la plus règlementée, ce qui pourrait laisser peu de place à une autorégulation. De plus, il paraît contraire au système de certification d'affirmer que le droit souple ne crée aucune contrainte. Pourtant il n'en est rien : l'entrée dans une telle démarche, même certifiée, résulte bien d'une adhésion volontaire. Précisément, en matière viticole, le lien entre initiative et sécurité juridique s'est traduit par l'adhésion volontaire des destinataires à des référentiels certifiés, ce qui permettait de guider les démarches éthiques individuelles tout en les faisant bénéficier des effets de la certification et de la labellisation.

Cela étant précisé, la RSE viticole est certainement plus large que la conversion en agriculture biologique ou l'adoption de référentiels certifiés de Responsabilité sociale et environnementale, ce qui justifie que soient non seulement envisagés ces dispositifs certifiés $(2,1,1)$, mais aussi les initiatives innommées et nombreuses de la filière, illustrations des possibilités nombreuses offertes par les sources juridiques mobilisées par la filière viticole dans son approche de la Responsabilité sociale et environnementale $(2,1,2)$.

\subsubsection{Les démarches certifiées}

La progression environnementale s'appuie sur deux éléments essentiels que sont la certification et la labellisation. Si l'on en veut une définition générale, la certification s'entend de la reconnaissance et du contrôle de conformité réalisés dans le cadre et par application de la normalisation technique. Plus précisément, il s'agit d'une attestation, après vérification, de la conformité d'un produit ou d'un service à certaines caractéristiques qui sont définies, ainsi que les modalités du contrôle du conformité, par un document technique, nommé référentiel, service assuré, à la demande des intéressés, par des organismes certificateurs indépendants de ces derniers qui sont habilités, sous le contrôle de l'Etat, par des instances d'accréditation [Vocabulaire juridique de l'association Henri Capitant, $\mathbf{v}^{\circ}$ Certification (7’̀me éd., Puf., 2005)].

Selon le comité français d'accréditation la certification est « une attestation délivrée par une tierce partie relative à des produits, des processus, des systèmes ou des personnes». Pour l'ISO il s'agit d' «une assurance écrite (sous la forme d'un certificat) donnée par une tierce partie qu'un produit, service ou système est conforme à des exigences spécifiques».

La certification atteste ainsi que le produit présente un certain nombre de caractéristiques définies dans un document de référence et auxquelles les consommateurs, voire plus généralement les clients, sont censés attacher une importance décisive.

Pour valoriser une démarche de certification, il est essentiel de labelliser le produit. Dans son acception juridique la plus large, le label est synonyme du terme «signe», et désigne à ce titre tout signe qui garantit aux utilisateurs la mise à disposition de produits ou de services répondant à des caractéristiques particulières régulièrement contrôlées. Cela désigne donc soit une marque collective soit un signe officiel de l'origine et de la qualité, soit une mention valorisante. En revanche, dans son acception juridique la plus stricte, il n'existe qu' un " label " officiel en agriculture, c'est le label rouge. La labellisation consiste dès lors à apposer un tel signe sur un produit. Le logo est quant à lui, selon le Code de la propriété intellectuelle, l'un des signes figuratifs susceptibles de représentation graphique susceptibles à ce titre de constituer une marque [CPI, art. L. 711-1]. 
Si dans un sens large la normalisation renvoie à la production d'une règle, contraignante ou non, destinée à diriger les comportements [V. G. Raymond, Droit de la consommation, $\mathbf{n}^{\circ} 206$ et $\mathbf{s}$., (Lexis-Nexis, $4^{\text {ème }}$ éd., 2017)], la normalisation appliquée aux référentiels de l'ISO ou de l'AFNOR obéit à une définition plus précise. Le décret n 2009-697 du 16 juin 2009 définit par exemple la normalisation comme «une activité d'intérêt général qui a pour objet de fournir des documents de référence élaborés de manière consensuelle par toutes les parties intéressées, portant sur des règles, des caractéristiques, des recommandations ou des exemples de bonnes pratiques, relatives à des produits, à des services, à des méthodes, à des processus ou à des organisations. Elle vise à encourager le développement économique et l'innovation tout en prenant en compte des objectifs de développement durable». L'ISO définit quant à elle ses normes comme des «documents qui définissent des exigences, des spécifications, des lignes directrices ou des caractéristiques à utiliser systématiquement pour assurer l'aptitude à l'emploi des matériaux, produits, processus et services».

Il s'agit ici de faire l'application d'une norme ne contenant pas en elle-même la garantie de son effectivité, car il faut que le professionnel y adhère. Il faudra enfin que l'entreprise, pour prouver la réalité de son adhésion, obtienne une certification ou une évaluation [Sur normalisation et certification, v. aussi E. Ayissi Manga, «La certification et le droit privé», Revue Juridique de l'Ouest, p. 257 et s. (200)]. La certification ou l'évaluation ne porte pas nécessairement sur le produit mais peut concerner l'entreprise. La norme ISO 14001 spécifie par exemple «les exigences relatives à un système de management environnemental pouvant être utilisé par un organisme pour améliorer sa performance environnementale et permet la mise en place d'un système de management environnemental qui permet l'amélioration de la performance environnementale, le respect des obligations de conformité et la réalisation des objectifs environnementaux». La norme ISO 14001, certifiée, n'est pas un signe de qualité du produit mais s'applique aux aspects environnementaux des activités, produits et services que l'organisme détermine, sans établir de critères spécifiques de performance environnementale. La norme ISO 26000 donne quant à elle «des lignes directrices aux entreprises et aux organisations pour opérer de manière socialement responsable». Cette norme, qui a pour particularité de contenir des lignes directrices et non des exigences ne se prête donc pas à la certification, mais elle peut être évaluée [V. par exemple les quatre niveaux de l'AFAQ : initial, progression, confirmé, exemplaire]. Quant à la norme Afnor NF-V01-007, elle permet la mise en œuvre d'un système de management de la qualité et de l'environnement de la production agricole et fournit ainsi un modèle pour la maîtrise des engagements réciproques entre les producteurs et leur organisme agricole pour répondre aux attentes des clients et parties intéressées.

Tous ces référentiels ont été adoptés et adaptés par la filière viticole ce qui a donné naissance à de très nombreux référentiels sectoriels nationaux ou régionaux tels que Terra Vitis, Viticulture durable en Champagne, AREA, VIVRE, Qualenvi, SME du vin de Bordeaux La norme ISO 14001 et son système de management environnemental a été privilégiée par l'interprofession viticole bordelaise. Six autres interprofessions viticoles (BIVB, InterOc, CIVC,
BIVC, CIVR, Bergerac) ont quant à elles approuvé -en partenariat avec l'AFNOR- un guide pour la mise en œuvre de la norme ISO 26000 en matière de viticulture.

Ces référentiels peuvent être reconnus équivalents à un niveau de certification environnementale de droit français [v. M. Degoffe, J.-Cl. Environnement et développement durable, Fasc. 3760, Règles de bonnes pratiques agricoles, $\mathbf{n}^{\circ} 38$ et s.]. Créée par la loi $\mathrm{n}^{\circ}$ 2010-788 du 12 juillet 2010 portant engagement national pour l'environnement, codifiée à la place de l'agriculture raisonnée à l'article L. 611-6 du Code rural et de la pêche maritime, précisée par les décrets $n^{\circ} 2011-694$ du 20 juin 2011 et n ${ }^{\circ}$ 20162011 du 30 décembre 2016, règlementée aux articles D. 617-1 et suivants du Code rural et de la pêche maritime, la certification environnementale s'articule selon trois niveaux. Pour pouvoir demander une certification environnementale, l'exploitation agricole doit atteindre un premier niveau d'exigence environnementale, considéré comme atteint dès lors que l'exploitant a réalisé un bilan démontrant que son exploitation satisfait aux exigences de l'article $93 \mathrm{du}$ règlement (UE) $\mathrm{n}^{\circ} 1306 / 2013 \mathrm{du}$ Parlement européen et du Conseil du 17 décembre 2013 relatives à l'environnement, au changement climatique, à la santé végétale et, si l'exploitation y est soumise au titre de ce règlement, aux bonnes conditions agricoles et environnementales des terres ; lorsque l'exploitant a réalisé une évaluation au regard du référentiel de deuxième niveau ou au regard des seuils de performance environnementale de troisième niveau. Ce premier niveau correspond à la mise en œuvre de bonnes pratiques agricoles [CRPM, art. D. 617-2]. La certification de deuxième niveau [CRPM, art. D. 617-3], dénommée «certification environnementale de l'exploitation», atteste du respect par l'ensemble de l'exploitation agricole des exigences environnementales figurant dans un référentiel établi par un arrêté du Ministre chargé de l'agriculture et du Ministre chargé de l'environnement [A. 20 juin 2011 portant application de l'article D. 617-3 du Code rural et de la pêche maritime et arrêtant le référentiel relatif à la certification environnementale des exploitations agricoles]. Ce référentiel prévoit 16 exigences qui permettent d'obtenir la certification environnementale. Le Code rural et de la pêche maritime prévoit ainsi que les démarches attestant le respect d'exigences équivalentes à celles définies à l'article D. 617-3, et dont la procédure de contrôle offre les mêmes garanties que celles fixées à la section 3 du présent chapitre, peuvent être reconnues en tant que certification totale ou sectorielle de deuxième niveau dénommée «certification environnementale de l'exploitation», par arrêté du Ministre chargé de l'agriculture, pris après avis de la Commission nationale de la certification environnementale mentionnée à l'article D. 611-18. C'est à ce niveau que se situent de nombreux référentiels, [https://agriculture.gouv.fr/certificationenvironnementaleliste-des-demarches-reconnues-par-le-ministere-de-lagricu lture]. C'est aussi à ce niveau que se situe l'agriculture raisonnée [Reléguées au second plan par la loi du 12 juillet 2010 portant engagement national pour l'environnement, les dispositions relatives à l'agriculture raisonnée résultent d'un décret du 25 avril 2002 pris en application de l'article 58 de la loi du 15 mai 2001 relative aux nouvelles régulations économiques]. Au $1^{\mathrm{er}}$ janvier 2018, environ 12000 exploitations possédaient cette certification de niveau II, soit environ $3 \%$ des 450000 exploitations 
agricoles françaises. Le troisième niveau (HVE) s'appuie sur des niveaux d'indicateurs à atteindre permettant de mesurer les performances environnementales des exploitations. Au $1^{\text {er }}$ janvier 2019 il concerne seulement 1518 exploitations, principalement viticoles.

La réglementation sur l'agriculture biologique [sur laquelle v. R. Romi et B. Lormeteau, J.-Cl Rural, fasc. 30, «Qualité des produits, Agriculture biologique»] relève quant à elle directement du droit de l'Union européenne [règlement $(\mathrm{CE}) \mathrm{n}^{\circ} 834 / 2007$ du conseil du 28 juin 2007 et son règlement d'application (CE) $n^{\circ} 889 / 2008$ de la Commission du 5 septembre 2008]. Elle permet au producteur d'apposer un logo sur le produit obtenu par un système de production agricole qui exclut l'usage d'engrais chimiques et de pesticides de synthèse, qui limite l'emploi d'intrants et qui respecte le bien-être animal et encadre strictement l'usage de médicaments. Cette gestion d'ensemble de la production favorise tout l'agrosystème, la biodiversité, les activités biologiques des sols et les cycles biologiques. En France, l'agriculture biologique et ses signes entrent dans le périmètre de la mission de l'INAO.

Le règlement 2018/848 du Parlement européen et du conseil du 30 mai 2018 relatif à la production biologique et à l'étiquetage des produits biologiques a été publié au JOUE le 14 juin 2018. Il abrogera le règlement $C E n^{\circ}$ 834/2007 du Conseil au 31 décembre 2020. Une seconde étape, celle de la négociation des actes d'application, d'achèvera en 2020 et la nouvelle règlementation entrera en vigueur le $1^{\text {er }}$ janvier 2021[L.- T. Mouflard, «Un nouveau règlement pour l'agriculture biologique : quel intérêt pour le secteur vitivinicole ?», Jus Vini, $\mathbf{n}^{\circ}$ 2, p. 22 et s. (2018)].

Le vin biologique existe depuis un règlement $n^{\circ}$ 203/2012 du 8 mars 2012 [V. J.-M. Bahans, «Vins biologiques et signes distinctifs», Vin, droit et santé, Deuxième millésime, p. 9 et s. (Les études Hospitalières, 2013)] les règlements précités ne contenant pas de dispositions spécifiques à la vinification. Pour l'essentiel, le vin biologique se résume à l'interdiction de certaines pratiques culturales et de vinification et à un abaissement du seuil de soufre. Il est porté par des signes officiels de qualité de l'agriculture biologique que sont le label biologique de l'Union européenne (eurofeuille), par le logo Agriculture Biologique (qui est aussi une marque) du droit français, ainsi que par la mention «vin biologique» règlementée par le droit de l'étiquetage [J. -M. Bahans, «Vins biologiques et signes distinctifs», préc., p. 16 et s.]. L'article 18 du règlement 2018 /848 prévoit que la commission est habilitée à adopter des actes délégués (annexe II, partie VI, point 3.2, en ajoutant des pratiques procédés et traitements œnologiques supplémentaires interdits, ainsi que l'annexe II, partie VI, point 3.3, qui n'interdit pas mais encadre certaines pratiques).

Moins contraignant qu'il n'aurait pu l'être [N. Hakim, «Lectures de la norme communautaire sur le vin biologique du 8 mars 2012», in N. Hakim et J.-M. Bahans (dir.), Le droit du vin à l'épreuve des enjeux environnementaux, (Féret, 2015)] et finalement vidé d'une partie de sa substance environnementale, le vin biologique a été dépassé, dans son exigence environnementale, par les vins biodynamiques et les vins dits «nature» [v. infra], ce qui montre aussi les limites des modèles construits par l'autorité publique, fruits de compromis politiques.
A l'inverse du vin biologique, le vin biodynamique est régi par des référentiels d'origine privée et par des marques privées de certification. La biodynamie concerne tant la production et la récolte de raisins que la vinification. Les utilisateurs de la marque doivent respecter à la fois le cahier des charges du label bio de l'Union européenne et le cahier des charges de la biodynamie. La biodynamie impose des prescriptions plus sévères par exemple en matière d'intrants, avec des seuils de soufre encore abaissés et une utilisation plus modérée du cuivre.

\subsubsection{Les autres dispositifs}

L'extrême règlementation viticole-justifiée par la protection des consommateurs, la régulation du marché et la défense des producteurs - a plutôt engagé les acteurs sur une éthique certifiée, parfois directement canalisée par la règlementation publique. A côté ou en complément de ces démarches certifiées les acteurs de la filière ont également adapté les démarches singulières des grands groupes. Comme l'indique le Conseil d'état le droit souple a vocation à se substituer au droit dur en l'absence de réglementation [Conseil d'état, Etude préc., p. 10] et donc dans les cadres laissés vides par la règlementation rigide de la matière viticole.

La RSE originelle, celle des multinationales, s'est fondée sur deux instruments : les Codes de bonne conduite et le reporting extra financier.

Dans le sillage des Codes de bonne conduite et autres chartes éthiques, la filière viticole utilise fréquemment la charte associative pour faire la promotion de certaines valeurs et unir autour d'un même projet.

La fonction publicitaire de l'éthique de l'entreprise est ainsi mobilisée, ce qui paraît parfaitement logique dans le cadre restreint posé par la loi Evin, les chartes et guides de consommation responsable s'étant multipliées au sein de la filière.

Les chartes associatives ont par exemple été utilisées par les producteurs de vin naturel pour structurer leur mouvement. Portés par une recherche d'épure, les producteurs de vin naturel poursuivent une démarche radicale via la diminution de la part d'intervention extérieure par la main de l'homme, de la machine ou par le recours aux intrants. On dit souvent que le vin naturel n'a pas d'existence juridique. C'est vrai au regard de l'ordre public viticole, mais c'est à nuancer si l'on prend en considération l'autorégulation associative. Le vin naturel, objet de droit naissant, pose à ce titre deux questions «naturelles» au juriste, celle de sa définition et celle de sa réglementation [L. Dawid, «Ethique du vin et «vide juridique»: le défi des vins naturels», in R.Raffray (dir.), L'éthique et le vin. Le droit comme levier d'une nouvelle qualité? Dr. et Patr. $\mathbf{n}^{\circ} 281$ (2018)]. Sur ces deux points, il est notable que la notion et le régime juridique du vin naturel devront s'épanouir dans le cadre dessiné par ses producteurs, l'INAO et la filière biologique, attentive à l'émergence du mouvement. De nombreuses pistes existent, de la structuration par une associationsituation actuelle - à la mise en place d'une certification ou la création d'un label. L'INAO est longtemps restée en position d'attente après avoir les bases d'une réflexion dans un communiqué de presse du 18 février 2016 mais le débat récurrent autour des sulfites montre que le match que jouent ces producteurs est déjà, sur le plan-là, bien engagé. 
Un rapport préparé a été enterré par l'INAO semblet-il sous la pression des représentants des appellations d'origine, ce qui montre que la viticulture différenciée peut aussi être une menace pour l'unité de l'appellation. L'association se dirigerait vers une marque privée.

Le contrat apporte également ses ressources pour la maitrise des approvisionnements, les maisons de négoce imposant à leurs fournisseurs des règles de traitement et valorisant l'apport de raisins et de vins bio ou produits sous certification environnementale [pour une illustration, à Cognac, v. Question écrite $\mathrm{n}^{\circ} 1942$ : Crédit d'impôt et certification «haute valeur environnementale» dans le secteur viticole, JO Sénat (9 novembre 2017)]. Ce recours aux procédés contractuels, caractéristiques de la maîtrise de la supply chain, illustre l'importation des ressources juridiques des grandes entreprises dans la filière viticole. On observera également le recours à la technique de cartographie des risques pour que coïncident traitement phytosanitaire et protection des voisins. A Bordeaux, une évolution sensible s'est enclenchée avec l'indisposition des élèves d'une école élémentaire après traitement. La réaction est venue de l'interprofession, qui a invité les utilisateurs à ne plus utiliser certains pesticides CMR (cancérogènes, mutagènes et reprotoxiques). Un atlas des zones sensibles a ensuite envoyé à plus de 900 viticulteurs concernés par un arrêté préfectoral du 26 avril 2016 fixant les mesures destinées à préserver les lieux et établissements sensibles accueillant les personnes vulnérables au risque d'expositions aux produits phytopharmaceutiques. Les dispositifs ont ensuite été repris par la loi Egalim du 30 octobre 2018, son article 83 laissant le soin aux parties prenantes d'autoréguler le traitement des zones en contact avec le voisinage, le préfet pouvant, à défaut d'autorégulation, en prononcer l'interdiction [CRPM, art. L. 253-8, III.].

La filière vin a longtemps souffert d'une communication lacunaire sur les actions menées et a choisi de refaire son retard. Cette communication, devenue essentielle, se réalise en droit économique par le reporting extra financier. Après avoir été invitées à intégrer au rapport de gestion des informations sur la manière dont la société prend en compte les conséquences sociales et environnementales de son activité, les grosses sociétés sont aujourd'hui tenues d'y insérer une déclaration de performance extra-financière qui comprend «(...) notamment des informations relatives aux conséquences sur le changement climatique de l'activité de la société et de l'usage des biens et services qu'elle produit, à ses engagements sociétaux en faveur du développement durable, de l'économie circulaire et de la lutte contre le gaspillage alimentaire, aux accords collectifs conclus dans l'entreprise et à leurs impacts sur la performance économique de l'entreprise ainsi que sur les conditions de travail des salariés et aux actions visant à lutter contre les discriminations et promouvoir les diversités (...)» [C. com., art. L. 225-102-1]. Cette communication a vocation à se généraliser à un grand nombre d'entreprises et d'institutions. Il entre d'ailleurs dans les missions des interprofessions d'encadrer de telles démarches et d'en restituer les résultats. Au-delà de ce cadre institutionnel les acteurs économiques se sont emparé de ce moyen de communication, alors même qu'ils n'y sont pas légalement assujettis, en profitant de sa fonction publicitaire et de l'élan qu'il permet de donner. La cave des vignerons de
Buzet a ainsi fait de son rapport développement durable une véritable publication (au sens propre du terme) qui restitue très exactement les actions menées au profit de l'environnement, des salariés ainsi que les améliorations en termes de gouvernance, dans une synthèse heureuse de gouvernance interne (comment l'entreprise est-elle dirigée ?) et externe (que faire pour les parties prenantes?) [Sur la gouvernance durable, v. C. Malecki, Responsabilité sociale des entreprises, Perspectives de la gouvernance d'entreprise durable, (LGDJ, Lextenso éditions, coll. Droit des affaires, 2014)].

\subsection{Le droit dur}

En droit économique, la RSE a fait une entrée remarquée dans le Code civil français à la faveur de la loi Pacte [LOI n ${ }^{\circ}$ 2019-486 du 22 mai 2019 relative à la croissance et la transformation des entreprises, JORF $\mathbf{n}^{\circ} \mathbf{0 1 1 9} \mathrm{du}$ 23 mai 2019 texte $\mathrm{n}^{\circ}$ 2] qui a modifié l'article $1833 \mathrm{du}$ Code civil. Une société doit aujourd'hui être gérée «dans son intérêt social, en prenant en considération les enjeux sociaux et environnementaux de son activité». En droit rural, l'article 48 de la loi Egalim prévoit, à l'horizon 2030, dans des conditions fixées par décret, une certification environnementale obligatoire pour tous les signes de qualité et de l'origine visés à l'article L. 640-2 du Code rural et de la pêche maritime. L'article 48 dispose ainsi qu' «(...) un décret fixe les conditions dans lesquelles, au plus tard le $1^{\mathrm{er}}$ janvier 2030, les signes d'identification de la qualité et de l'origine mentionnés au $1^{\circ}$ de l'article L. 640-2 du code rural et de la pêche maritime intègrent dans leurs cahiers des charges les dispositions pour que les exploitations concernées répondent aux exigences prévues pour faire l'objet de la certification prévue à l'article $L$. 611-6 du même code (...)» .

Le texte illustre la place prise par les considérations environnementales et la préservation de la biodiversité en agriculture, sans que celles-ci ne soient cantonnées aux agricultures différenciées, telles que l'agriculture biologique, seul signe officiel d'identification de la qualité environnementale. Face au défi environnemental la segmentation au sein des signes de qualité, entre qualité environnementale (le bio), qualité supérieure (le label rouge), «signes de qualités du produit»et qualité liée à l'origine (AOP et IGP), «signes de qualité du terroir» [J.-P. Combenègre, ouvrage préc.] apparaît subitement comme inadaptée. La loi Egalim cherche ainsi à revoir à la hausse les exigences environnementales sans bouleverser les équilibres construits autour des différents signes de qualité du produit. Cette ambition implique la certification environnementale de tous les producteurs exploitant sous signe de qualité et de l'origine.

\subsubsection{Les signes concernés}

Les signes de qualité et de l'origine de l'article L. 640-2 du Code rural et de la pêche maritime présentent des caractères communs. Ce sont tous des signes de qualité du produit, placés sous la tutelle de l'INAO, chargé de mettre en œuvre les dispositions législatives et réglementaires relatives à ces signes (CRPM, art. L. 642-5), mais qui répondent à des objectifs distincts, énoncés à l'article L. 640-1 du même code. L'appellation d'origine et l'indication géographique protégée expriment 
une qualité du produit liée à l'origine, la spécialité traditionnelle garantie exprimant quant à elle une qualité liée à la tradition. Le Label Rouge atteste de son côté d'une qualité supérieure. Seule l'agriculture biologique garantit donc, au sein de ces signes officiels, l'exigence de qualité environnementale.

Placer les démarches agroécologiques des producteurs sous signe de qualité et de l'origine dans le giron de la certification environnementale obéit à la logique de la certification sans déroger aux règles gouvernant les SIQO. Certifiant l'entreprise et non le produit la certification environnementale peut se combiner avec les signes de qualité et de l'origine du produit, y compris l'agriculture biologique, qui bénéficient de leurs propres systèmes.

Lors des discussions qui ont précédé le vote de la modification de l'article L. 611-6 du Code rural et de la pêche maritime une volonté de labelliser l'agroécologie par utilisation du label «Haute Valeur Environnementale» a été fortement exprimée. En outre l'article 24 de la loi, consacré à la restauration collective, fait mention, à compter du 1er janvier 2030, des produits $\ll(\ldots)$ issus des exploitations ayant fait l'objet du plus haut niveau de certification prévu à l'article L. 611-6 (...)». C'est enfin à ces seules exploitations que l'article 82 de la loi réserve l'expérimentation sur le traitement phytopharmaceutique aérien.

Il apparait toutefois trop ambitieux d'exiger une certification de niveau 3 de droit français et il n'est pas exclu que l'on se dirige vers une certification intermédiaire de niveau 2 ou entre les niveaux 2 et 3 . Lors des débats sur l'obligation de certification environnementale imposée aux SIQO, les parlementaires ont effet indiqué vouloir laisser le temps au Ministre de dialoguer avec la profession (avec un décret établi d'ici à 2021). En se référant à la certification de l'article L. 611-6, sans autre précision, le texte entretient donc l'incertitude, jusqu'à la publication du décret, sur le niveau de certification qui sera exigé, et laisse ainsi la place à une progression qui ne peut se réaliser que par paliers.

\subsubsection{Le recours au cahier des charges}

L'article L. 622-22 du code rural prévoyait déjà que «l'organisme de défense et de gestion peut élaborer une charte de bonnes pratiques contenant des dispositions de nature à préserver certaines caractéristiques environnementales de son terroir ou des dispositions spécifiques en matière de bien-être animal». Il est également indiqué que «le respect de cette charte n'est pas une condition d'obtention du signe d'identification de la qualité et de l'origine». L'article 48 de la loi Egalim renvoie également au cahier des charges pour que les exploitations concernées répondent aux exigences prévues pour faire l'objet de la certification environnementale. La technique du cahier des charges est commune aux signes d'identification de qualité et de l'origine [CRPM, art. L. 641-1 et s] et en constitue le support juridique essentiel [F. Robbe, «Le cahier des charges, support juridique des signes de qualité», RD rur. $\mathbf{n}^{\circ} 396$ (Octobre 2011)]: présent à toute étape de la reconnaissance, de la gestion et de la protection du signe qu'il soutient il garantit l'homogénéité de la règlementation des signes de qualité sur le territoire [F. Robbe, art, préc.]. Il apparaît tout à la fois comme le moyen de définir le produit, d'informer le consommateur quant à ses caractéristiques et de garantir sa qualité, son authenticité-et, pour les AOP et IGP, son origine [N. Olszak, «Les nouveaux règlements européens sur les appellations d'origine et indications géographiques protégées et les spécialités traditionnelles garanties»:RD rur. 2006, étude 19] - et assure la valorisation des produits agricoles [F. Robbe, art. préc., $\left.\mathrm{n}^{\circ} 14\right]$. Ainsi, en application du droit de l'Union européenne, le Code rural et de la pêche maritime [CRPM, art. R. 641-12] dispose que le cahier des charges «décrit la spécificité du produit, définit ses conditions de production, de transformation et éventuellement de conditionnement et d'étiquetage, précise s'il y a lieu l'aire et les zones dans lesquelles les différentes opérations sont réalisées et indique les principaux points à contrôler ainsi que leurs méthodes d'évaluation» L'INAO avait déjà engagé le chantier de l'insertion de mesures agroécologiques dans les cahiers des charges des indications géographiques viticoles, en préagréant des mesures qui pourraient ensuite être insérées dans les cahiers des charges, décision qui avait été suivie de la publication d'un guide de l'agroécologie en agriculture confectionné avec l'Institut français de la vigne et $\mathrm{du}$ vin. Cette intégration, volontaire $\mathrm{du}$ côté des ODG mais contraignante pour les producteurs, constituait déjà une évolution majeure en inscrivant la qualité environnementale dans le processus «de droit commun» de valorisation collective de la production agricole [R. Raffray, «Une RSE innommée au service des démarches éthiques de la filière vin», préc].

Il y a quelques années, un auteur a écrit, à propos de l'élaboration des cahiers de charges, qu'elle était déterminée par un «dirigisme teinté de démocratie» [F. Robbe, art. préc]. Le même constat s'impose à propos du durcissement de la règlementation poursuivie par la loi Egalim. Obliger à une certification environnementale constitue une démarche nouvelle car les itinéraires environnementaux étaient jusqu'à aujourd'hui spontanément empruntés, selon le modèle d'une éthique encouragée par les pouvoirs publics. Ce renforcement apparaissait nécessaire [P.-E. Bouillot, ouvrage préc., $\mathrm{n}^{\circ}$ 268]. car l'engagement volontaire, moteur d'une agriculture différenciée, a comme ailleurs montré ses limites [P.-E. Bouillot, ouvrage préc., n 206 et s.]. Ce cheminement, du droit souple ou mou au droit dur, rencontré sur le terrain d'élection du droit souple qu'est le droit des grandes entreprises, trouve aussi sa place en matière agricole. L'agriculture ne peut échapper aux dispositifs juridiquement contraignants, la contrainte économique s'exerçant déjà par les stipulations conditionnant les relations contractuelles au respect de certaines pratiques. Le Conseil d'état identifie quatre fonctions du droit souple [Conseil d'état, étude préc., p. 86 et s.]. Il peut notamment préparer un recours au droit dur, en habituant progressivement les acteurs. En redynamisant de la sorte un label public, la loi réaffirme aussi la dimension publique et collective de la production de qualité.

\section{La notion de qualité}

L'évolution des enjeux qualitatifs du vin s'exprime historiquement par le passage de la qualité de vin (qu'estce qu'un vin ?) à la qualité du vin (qu'est-ce qu'un bon vin ?) [V. notamment O. Serra, Le législateur et 
le marché vinicole sous la Troisième République, thèse, Bordeaux (2012)]. La qualité de vin renvoie aux enjeux d'une première définition légale du vin, la fraude ayant contraint le législateur à définir le vin en combinant les critères de la méthode - la fermentation alcooliqueet du matériau-le raisin frais. La qualité du vin en est complémentaire et en permet une hiérarchisation qualitative [J.-M. Bahans et M. Menjucq, Droit de la vigne et du vin. Aspects juridiques du marché vitivinicole, $2^{\text {ème }}$ éd. LexisNexis-Féret, p. 72 et s.] Si la qualité liée à l'origine domine la hiérarchisation qualitative, la notion de qualité, ne semble pas rétive à l'intégration $\mathrm{du}$ progrès environnemental. La qualité est en effet protéiforme et évolutive. Selon E. Peynaud et J. Blouin [Le Goût du vin, p. 192 et s. (Dunod, 5 ème éd., 2013)], les qualités du vin sont plurales : les auteurs distinguent la qualité viticole, les qualités analytiques, les qualités hygiéniques et alimentaires, les qualités administratives ou formelles et les qualités économiques et culturelles. En outre, selon les mêmes auteurs «la qualité est le résultat d'une rencontre entre le vin, avec ses caractéristiques, et le consommateur, avec ses préférences, sa culture, ses conditionnements du moment». Pour le juriste la première qualité du vin est certainement organoleptique, c'est-à-dire telle qu'elle est envisagée par le prisme des récepteurs sensoriels exprimant ainsi le terroir [Sur la notion, v. C. Le Goffic, L'appellation d'origine, reconnaissance juridique du concept géographique de terroir, $R D$ rur. $\mathrm{n}^{\circ} 358$, déc. 2007, étude 37] qui a présidé à son élaboration. Elle se confond peut-être, dans cette perspective, avec la typicité, en ce sens que le vin sous indication géographique est un vin caractéristique de son terroir.

Le contexte de production et d'élaboration durable a fait naître des questionnements complémentaires, rattachables à ce que l'on peut identifier comme une qualité sociale et environnementale. Il est par exemple remarquable des classements des crus exigent aujourd'hui, dans le dossier de candidature au classement, la preuve d'une certification environnementale (c'est le cas pour le classement des crus bourgeois du médoc).

Cette nouvelle qualité peut potentiellement s'intégrer au concept de terroir qui exprime l'interaction entre un milieu et des pratiques. Pour le juriste comme pour l'agronome, le terroir est en effet «un concept qui se réfère à un espace sur lequel se développe un savoir collectif, des interactions entre un milieu physique et biologique identifiable et les pratiques vitivinicoles appliquées, qui confèrent des caractéristiques distinctives aux produits originaires de cet espace» [Rés. OIV/Viti 333/2010].

Les signes de qualité environnementale, complémentaires des signes de l'origine ont certainement joué un rôle de relais entre la qualité liée à l'origine et la qualité telle qu'elle est perçue dans une démarche RSE, ne seraitce que parce que l'agriculture biologique est placée sous la surveillance de l'INAO et que la biodynamie obéit à un régime de certification proche de certaines démarches RSE, mais aussi parce que le vin biologique, né avec le règlement $\mathrm{n}^{\circ} 203 / 2012$ du 8 mars 2012 s'est révélé moins contraignant qu'il n'aurait pu l'être et a été dépassé, dans son exigence environnementale et d'authenticité, par les vins biodynamiques et les vins dits «nature» ou «naturels».

La force du droit qui vient d'en bas, dans un rôle de substitut ou d'inspirateur, ne doit jamais être sous-estimée.

Cette évolution de la qualité ne se fera pas sans de nouvelles clarifications. La généralisation des certifications environnementales est à ce titre susceptible de créer une ambiguiité quand l'on prend en compte ce qui est ainsi valorisé : est-ce le produit ? Est-ce l'entreprise ?

En matière de certification environnementale, la confusion est entretenue par la création des deux marques collectives : une marque collective «Haute Valeur Environnementale» qui a pour objet d'identifier auprès du public les exploitations agricoles certifiées de «Haute valeur environnementale»; une marque collective «Issu d'une exploitation de Haute Valeur Environnementale», réservée aux produits. La confusion sera accentuée par l'insertion des mesures agro écologiques dans le cahier des charges.

Trois voies pourraient être empruntées.

La première serait de faire de cette qualité environnementale l'une des facettes de cette qualité/typicité. C'est d'ailleurs sur ce terrain que se place le discours, par exemple, de la biodynamie et des vins naturels. La deuxième serait de considérer cette qualité environnementale comme annexe et complémentaire de la qualité / typicité. On pourrait enfin, en troisième voie, envisager de distinguer, parmi les apports de la viticulture différenciée, ceux qui relèvent de la stricte qualité sociale et environnementale et ce qui relèvent de la typicité dès lors que cela a une influence sur le goût. 